\title{
Entretien avec Bruno Tackels : « Le rater ce n'est pas qu'un événement, c'est un mode d'être et de créer »
}

\section{Bruno Tackels et Estelle Baudou}

\author{
(2) OpenEdition \\ Journals \\ Édition électronique \\ URL : https://journals.openedition.org/agon/8384 \\ DOI : $10.4000 /$ agon.8384 \\ ISSN : 1961-8581 \\ Éditeur \\ Association Agôn
}

Référence électronique

Bruno Tackels et Estelle Baudou, «Entretien avec Bruno Tackels : " Le rater ce n'est pas qu'un événement, c'est un mode d'être et de créer » », Agôn [En ligne], 9 | 2021, mis en ligne le 12 janvier 2022, consulté le 17 janvier 2022. URL : http://journals.openedition.org/agon/8384 ; DOI : https:// doi.org/10.4000/agon.8384

Ce document a été généré automatiquement le 17 janvier 2022.

Association Agôn et les auteurs des articles 


\title{
Entretien avec Bruno Tackels : « Le rater ce n'est pas qu'un événement, c'est un mode d'être et de créer »
}

\author{
Bruno Tackels et Estelle Baudou
}

ESTELLE BAUDOU : Avec la crise sanitaire, on peut dire qu'on a raté une saison, autant pour les spectateurs que pour les artistes. Une saison était programmée et on n'a pas pu assister. J'avais donc envie de démarrer par cette question-là : qu'est-ce que c'est que rater un spectacle parce qu'on ne l'a pas vu?

BRUNO TACKELS : Le rendez-vous manqué. Ce serait ça, au fond. Le théâtre, c'est un rendez-vous. Ce sont des gens qui, de leur lieu d'origine, se déplacent vers un lieu pour s'agréger en une communauté qui va faire le spectacle. Effectivement, si ces gens ne viennent pas, il n'y a pas cette agrégation et donc le rendez-vous est manqué. Rater un spectacle, c'est déjà par exemple se tromper de théâtre. Se tromper d'horaire. Ça nous arrive à toutes et tous. C'est une expérience terrible : soudain se rendre compte qu'on n'est pas devant le bon théâtre ou dans la bonne salle du théâtre, quand le théâtre a plusieurs lieux. Je trouve que c'est un sentiment vertigineux et très métaphysique. Ça dure un instant, après on peut vite courir pour essayer de rattraper le train en marche. Mais c'est un rendez-vous manqué. C'est une espèce de blanc qui s'installe, l'effacement qui s'installe. Là, depuis le 17 mars, on a l'impression d'un gros bruit blanc qui s'est installé dans le monde de la création, et dans celui de la scène en particulier.

E.B. : Rater un spectacle, c'est aussi ne pas avoir pris le temps d'y aller. On peut dire : "moi, je ne l'ai pas vu, je l'ai raté. »

B.T. : Oui, c'est vrai, c'est une phrase qu'on entend souvent: « je l'ai raté, celui-là ». Ça montre qu'il y a une connexion entre le simple fait physique du non-rendez-vous et le ratage justement. Ça me paraît vraiment important cette notion de rendez-vous. Comme le dit Claude Régy, pour rentrer dans un spectacle, il faut se donner rendezvous avec soi-même. Il faut déjà être soi-même disponible. On ne peut pas être au spectacle et à autre chose. Malheureusement, on voit bien que c'est de moins en moins vrai. L'arrivée de la technologie dans les salles de spectacle a beaucoup 
perturbé ce rendez-vous. Beaucoup de metteurs en scène essayent de travailler avec ça ou de lutter contre ça. Mais on ne peut pas être au théâtre et ailleurs en même temps. C'est la définition même du théâtre. Et pourtant la technologie produit ça. Structurellement, elle nous amène toujours dans cette espèce de dédoublement $\mathrm{du}$ temps où on est à la fois à une chose et à une autre. C'est-à-dire qu'on a toujours l'impression qu'on a cette vie ici et maintenant, et une autre vie qui est comme en parallèle. Ce fameux concept de second life.

E.B. : Et pendant cette crise que nous traversons, les spectacles n'ont finalement plus eu lieu que dans cette seconde vie.

B.T.: On a eu en effet ce mouvement de rabattement. C'est quand même très étonnant. Quand on parlait de captation, il y a encore six mois, c'était vraiment un sujet inabordable, inacceptable, ringardisé d'emblée. Bien sûr, la société de production audiovisuelle La Compagnie des Indes ${ }^{1}$ a montré qu'on pouvait faire un vrai travail avec des outils adéquats, avec de l'argent, une production et que cela pouvait vraiment générer des choses formidables. Mais c'est le seul exemple. On n'a vu aucun CDN, par exemple, s'en emparer. Il y a tout un argumentaire pour justifier cela : la fameuse présence du théâtre. Mais c'est ne pas vouloir aborder la question.

E.B. : Comme dans d'autres domaines, pensons au télétravail par exemple, la crise a eu un effet accélérateur sur ce sujet, au moins provisoire.

B.T. : Soudain, on a une bascule incroyable. En quelques mois, tout le monde s'est mis à montrer des captations. Ce qui veut donc dire que ce qui était considéré, encore il y a six mois, comme n'ayant aucune existence artistique s'est mis à en avoir une, grâce à Internet. Imaginons que cette crise sanitaire ait eu lieu en 2002. Qu'est-ce qui se serait passé ? On se serait peut-être envoyé des cassettes VHS. On aurait pu envoyer des revues à des abonnés. Mais comme nous sommes en 2020, soudain, la vie du théâtre est entrée dans cette seconde vie d'Internet et s'y est déployée, avec plutôt du succès. C'est une jolie manière de faire réapparaître une mémoire du théâtre. Moi ce qui me frappe beaucoup, c'est qu'on n'a pas saisi Internet comme le lieu du rendezvous au présent. À quelques exceptions près. Pourquoi ? Je n'ai pas la réponse, mais on voit bien qu'en France ça n'a pas eu lieu. Je dis "en France " parce que ici, en Colombie, il y a des metteurs en scène qui se sont emparés de l'idée qu'à $20 \mathrm{~h}$ ils seraient présents.

E.B. : Si le rendez-vous est raté, ce serait donc aussi parce que le présent n'a pas été investi par les artistes?

B.T. : Il y a d'abord eu une sidération. Et il y a eu très vite la prise de conscience que déjà les conditions de possibilité de l'acte théâtral étaient menacées pour longtemps. Il y a eu tout ce débat sur l'intermittence. Il est nécessaire. Malheureusement, c'est un débat qu'on aurait aimé régler en 2003, qu'on n'a pas réglé et qu'on traine. Cela empoisonne la vie artistique et culturelle depuis près de 20 ans. Fort heureusement, cette question est remise sur la table. Pour autant, cela n'a pas permis aux artistes d'être libres pour réfléchir à tout ça et pour agir, pour donner rendez-vous.

E.B. : Et qu'est-ce que ce rendez-vous raté révèle finalement de la situation des arts de la scène en France tant au niveau artistique qu'aux niveaux économique et politique?

B.T.: Elle révèle d'abord que le système est extrêmement vigoureux puisque la structuration du champ culturel en France est absolument unique. Il y a une espèce de machine extrêmement puissante qui s'est mise en route, qui s'est amplifiée et qui s'est aussi sédimentée. C'est une machine par couches : les centres dramatiques, les 
scènes nationales, les scènes conventionnées. Ça fonctionne par couches et donc certainement pas de manière organique. Et ça fonctionne! Mais soudain apparaît très clairement ce que Mathias Langhoff signalait dans un rapport pour le TNB: «Pourquoi transformer un théâtre qui fonctionne bien? Parce qu'il fonctionne bien. » Tout le système est en train de s'interroger lui-même.

Il y a trop d'impensés, trop de questions taboues, trop de choses qu'on a mises sous le tapis. Le libéralisme est entré entièrement dans le monde du théâtre. En effet, les mots mêmes de production et diffusion sont des mots qui n'ont rien à voir avec l'acte artistique. Quand Hubert Gignoux prend la direction du centre dramatique de l'est à Strasbourg et invente ce lieu (1957), la notion qui fonde son travail, c'est la tournée. $\mathrm{Au}$ sens littéral. On monte des tréteaux, on décharge le matériel en camionnette, et les actrices et les acteurs sont partie prenante de ce geste. Et cette notion de tournée n'a rien à voir avec ce qu'en font des metteurs en scène aussi talentueux que Joël Pommerat ou Julien Gosselin. La notion même de tournée est aujourd'hui vidée de son sens premier. Ce n'est pas pour dire que leur travail n'est pas juste. Ce travail a son sens, il est légitime et nécessaire, mais il n'est pas suffisant. Il ne doit pas empêcher la prise de conscience qu'on s'est un peu éloigné des objectifs et qu'il va falloir travailler autrement, réinventer. C'est là que malheureusement on peut rater beaucoup de choses parce qu'on peut avoir beaucoup de rêves, beaucoup de désirs.

\section{E.B. : Pourriez-vous donner un exemple de cette dynamique?}

B.T. : Le Festival d'Avignon. Si vous prenez le projet tel qu'Olivier Py l'a rêvé et si vous regardez ce qu'aurait dû être le Festival d'Avignon sur l'édition 2020, il y a une énorme différence. Il ne faut pas juger le projet ou sa non réalisation mais il faut réfléchir aux raisons pour lesquelles ça n'a pas été possible. Pourquoi y a-t-il tant de pistes évoquées par Olivier Py qui ne sont pas possibles? L'allongement du temps de diffusion d'un spectacle, par exemple. Un spectacle qui joue dans n'importe quelle scène nationale deux semaines pleines, du mardi ou samedi, ça n'existe plus. Et ça me paraît absolument terrifiant. Comment peut-on construire un public dans ces conditions? Il y a à Avignon ce problème bien connu que le jour où on ouvre la billetterie, il n'est déjà plus possible d'avoir une place. On a organisé les conditions de ratage du spectacle et le rendez-vous est manqué d'emblée. Comment est-ce qu'on défait ça ? Je n'ai pas la réponse. C'est une machinerie tellement complexe qu'il faut sans doute prendre des décisions radicales et je pense que la situation est propice à cela. Par exemple, à Avignon, il y a beaucoup trop de spectacles. Il pourrait y avoir une programmation avec 8 spectacles ou 12 spectacles.

E.B. : Est-ce qu'un des outils de cette réinvention ne pourrait pas être de renouveler le droit au rater et au raté ? C'est-à-dire au rater comme processus, l'expérimentation artistique, et au raté comme résultat? Ce droit au rater et au raté, dans ce que vous décrivez, il a été dénié aux artistes autant par les mécanismes de production que par les mécanismes de sélection et les enjeux de programmation.

B.T. : Le mot de sélection est un mot-clé. Le champ théâtral est constitué de cercles concentriques avec cette idée qu'on démarre son projet de compagnie au bord externe. Si on est très ambitieux, le but est d'intégrer le premier cercle et d'être un jour directeur d'un théâtre national. Cette logique des cercles concentriques se produit par sélection. Je pense que la sélection est un autre venin libéral du monde théâtral. On ne devrait absolument jamais sélectionner un artiste: le faire entrer dans un centre dramatique par ce processus de sélection - les fameuses short lists - 
c'est une aberration. C'est mettre en compétition des artistes les uns contre les autres, c'est les obliger à mettre toute leur énergie dans ce processus de sélection pour réussir - pour ne pas rater la sélection justement. Et ce n'est absolument pas ça l'ADN d'un artiste. On pourrait dire que dans le monde des arts visuels, le monde du marché oblige à ça. Mais même pas ! Parce que dans le monde du marché, l'artiste est en lien direct avec les acheteurs, un galeriste, un collectionneur, un curateur. D'une certaine manière, tous ces gens le sélectionnent mais pour faire son travail. Si un collectionneur choisit une œuvre, il rend possible le travail d'un artiste, mais le travail est fait. Or quand un artiste du monde du spectacle fait dans une année trois candidatures pour trois CDN et qu'il y passe des mois, il n'a toujours pas commencé son travail. Ça veut dire que l'on pose comme un geste réussi le fait d'accepter que son temps ne soit pas consacré à son activité artistique. C'est effrayant. C'est finalement programmer le ratage puisque c'est programmer l'impossibilité du travail.

Il faudrait cesser de sélectionner. C'est l'artiste qui se sélectionne, car c'est lui qui décide d'être artiste. C'est une décision suffisamment noble et difficile pour qu'on la respecte. Et si quelqu'un doit encore sélectionner, c'est le public. Aujourd'hui, c'est lui qu'il faut remettre en avant. Ce sont les spectateurs qui doivent avoir le dernier mot, pas la short list, pas les jurys. Ce sont eux qui sont déterminants car ce sont eux qui décident si c'est raté ou pas. La question de fond est la suivante : qui dit que c'est raté ? Quelle est l'instance qui juge et qui est légitime pour énoncer le ratage in fine? C'est le public! Et combien de fois on a vu ces cas de figure pathétiques où la presse est unanime - « c'est un raté » - mais où le public va ensuite plébisciter le spectacle. Parfois le lendemain ou le surlendemain de la première. C'est arrivé à Vitez. Le Soulier de satin, qu'il présente en 1987 à Avignon, était un raté au soir de la première. Vitez fait partie de ces gens qui ont besoin de beaucoup de temps et le processus d'Avignon est redoutable, on le sait. Donc le spectacle n'était pas prêt, mais il a ensuite été un des plus grands succès du festival d'Avignon voire de l'histoire du théâtre. Aussi les conditions de possibilité du jugement sont importantes : qui donne le droit de juger? Qui fabrique l'histoire du théâtre? Qui fabrique le jugement sur le théâtre ? Et quelles sont les instances qui permettent de déjouer ça?

E.B. : Le problème est donc que l'on se retrouve avec des institutions qui, par les processus de sélection et en particulier les appels à projets, décident de ce qui est raté ou pas avant même que ça existe.

B.T. : L'appel à projets est une chose terrifiante. On vous demande d'écrire un projet, de décrire ce que vous allez faire alors que vous ne l'avez pas fait. Le seul que je connaisse qui a su déjouer ça dans l'institution théâtrale, c'est François Tanguy avec le théâtre du Radeau à la Fonderie. Il a imposé l'idée que le projet c'était juste un titre, qui pouvait d'ailleurs changer. François Le Pillouër le savait très bien, lui qui faisait partie de ces importants producteurs qui ont contribué à la libéralisation du paysage et qui l'a fait avec beaucoup de talent, à l'écoute des artistes. Quand il allait voir François Tanguy dans la banlieue de Rennes, au campement, il savait très bien qu'il allait sortir de cette réunion - qui pouvait durer deux, trois, quatre heures avec... un titre. Il l'assumait, il savait qu'il ne pouvait pas demander plus à François Tanguy. C'était une impossibilité logico-philosophico-artistique. Une impossibilité humaine aussi car s'il avait mis François Tanguy sous pression, il se serait effondré ou enfui. 
On a l'impression que c'est une exception ou un point limite alors que ça devrait être la posture de tout artiste. Tout artiste pense comme François Tanguy mais soudain on s'est mis à penser autrement et à intérioriser l'obligation du projet. Ce geste est un non-sens. Quand on est devant un artiste, on doit lui faire confiance. La subvention publique doit se faire sur une base de confiance. Or, le mot employé est le mot de tutelle. Ce qui montre qu'on ne fait pas confiance aux artistes. La tutelle est là pour vérifier que l'artiste ne déborde pas. Mais un projet artistique fort, c'est un projet en excès, c'est un projet qui dépasse la mesure, qui va au-delà de l'imaginable. Jean Vilar, quand il investit la cour du Palais des papes, ce n'est pas imaginable. Quand Vitez monte Le Soulier de Satin, huit heures de spectacle, ce n'est pas imaginable. Quand Anatoli Vassiliev essaye de réussir ce pari fou de faire travailler les acteurs de la Comédie Française ${ }^{2}$ selon une méthode qui n'est absolument pas la leur - et qui ne peut donc que produire un ratage - on est dans la démesure absolue. On est dans l'impossible rendu possible. L'artiste est toujours dans ce franchissement des limites, il fait des choses qui ne sont pas permises. Cette logique institutionnelle de la tutelle est terrifiante: accepter de dialoguer avec des tutelles, c'est accepter qu'on est irresponsable au sens moral. Et oui, l'artiste est irresponsable au sens où il n'a à répondre qu'à lui-même. Tadeusz Kantor ne répondait qu'à lui-même. Imaginez Kantor répondre à un appel à projets! C'est quelque chose qu'il faut réformer profondément. Je ne dis pas que l'appel à projets ne peut pas avoir du sens dans certains cas mais la façon dont il s'est généralisé me paraît toxique. Ça fait que l'énergie de l'artiste est dévoyée, mise au service de quelque chose qui n'est pas son travail. Son travail c'est le plateau, c'est le laboratoire, c'est l'école. Il faut lui rendre sa liberté.

E.B. : Alors le plateau, le laboratoire, l'école, ce seraient précisément les espaces du rater.

B.T. : Du droit au rater !

E.B. : Et quel serait alors l'espace-temps idéal du rater comme espace de répétition ou de création?

B.T. : Grotowski disait : « Je ne comprends pas, en France, vos deux mois de répétition (je parle des années 1980). Le travail de répétition, c'est ou deux jours ou deux ans. » C'est le «vite-fait bien-fait» de Vitez: on tente, on pose un geste. Ou alors on est dans le dépliement d'un geste interminable. C'est ce qui a fait aboutir Grotowski à l'idée qu'il n'y a plus de spectacle car il n'est plus possible de couper ce flux continu. Il n'est plus possible de convoquer les spectateurs parce que le temps de la recherche est interminable.

E.B. : Est-ce que le laboratoire comme espace-temps du rater serait un espace infini sur un temps infini?

B.T.: Oui. Et je dirais que le problème de la recherche en art, c'est qu'on attend du laboratoire qu'il produise. Or je pense qu'un laboratoire, on ne sait pas ce qu'il va produire ni quand, ni où, ni même s'il va produire. Le laboratoire c'est une matrice dont les résultats ne viendront peut-être que vingt ans plus tard quand l'ensemble des membres de ce laboratoire ne se reconnaîtraient même plus dans la vie ordinaire. Et cet enchaînement-là ne se réduit à aucun appel à projets, à aucune formalisation. L'enseignement et la recherche, c'est de la pollinisation, c'est une plante en train de sécher et les graines partent au vent. Des insectes, des papillons de nuit, des abeilles vont venir et toute une chaîne va se mettre en place qui va assurer le cycle vital. Cette chaîne de pollinisation ne correspond à aucun algorithme. 


\section{E.B. : Elle ne s'évalue pas.}

B.T. : Il faut accepter la modestie de l'évaluation. L'évaluation est très relative, elle doit donc être très modeste. Dans le système de l'appel à projets, les évaluateurs sont plus importants que les candidats. Au ministère, j'ai beaucoup lutté pour que les membres des jurys soient payés. Mais, quand on y pense, c'est le monde à l'envers. C'est assumer l'idée qu'on octroie de l'argent à des gens qui vont évaluer des gens qui eux-mêmes n'auront probablement pas d'argent. C'est quand même une logique insensée ! Alors, oui, ça donnerait du travail à des artistes. En fait ça donnerait cette perversité : on engagerait des artistes dans les jurys, ce qui financerait un travail que, par ailleurs, ils n'arriveraient pas à financer. C'est l'aberration libérale greffée sur l'économie théâtrale.

Ici, en Colombie, on a un concept très puissant pour dire cette dérive libérale : c'est l'économie orange. On s'appuie pour cela sur quelques exemples historiques et en particulier les consoles de jeux Nintendo. Le modèle est donc le suivant: le geste créatif - dans cette théorie, le mot art a été supprimé - génère de l'argent de manière exponentielle. L'injonction de l'économie orange consiste à dire aux artistes qu'on ne va pas financer leur création mais que l'on va faire en sorte qu'elle génère de l'argent. Le mot créatif est un adjectif qu'on met partout: sur un projet, une question, une entreprise. On est dans le créatif partout et dans l'art nulle part. C'est un hold-up. Évidemment, 90\% des artistes colombiens n'entrent pas dans ce schéma et, par conséquent, ils se retrouvent hors-champ.

E.B.: Alors l'espace-temps du raté est forcément extérieur à l'économie libérale. Le libéralisme fait du raté la condition de la réussite en écartant les uns pour ne sélectionner qu'une minorité. Est-ce que les formations artistiques pourraient être préservées de cela? Qu'est-ce que serait une pédagogie du raté en art et en particulier dans la formation de l'acteur? Et quelle serait cette école qui ouvrirait l'espace-temps du raté ?

B.T.: Ce serait déjà la suppression des concours. La notion de concours est intimement liée à celle d'appel à projets. On a vu le milieu artistique vouloir se modeler sur la logique des grandes écoles. Les écoles nationales de théâtre ne sont pas autre chose. Il leur a longtemps manqué les classes prépa. Je salue l'idée qu'elles existent aujourd'hui et de très haut niveau, car dans ce contexte elles sont salutaires. Mais ça reste un monde d'une sélection absolue. Moi j'ai passé l'agrégation de philo et je l'ai réussie. Je peux en parler tranquillement. Il y avait 1200 candidats, je crois, et 35 postes. On voit la disproportion incroyable entre le nombre de postulantes et postulants et le nombre d'élus. On est dans l'élection au sens théologique. Une fois qu'on est élu, il n'y a plus rien à faire au fond. Les écoles balisent un champ du connu alors qu'il faudrait pouvoir s'exposer à l'inconnu et à l'inconnu du ratage. Ça permettrait d'éviter que les élèves, quand ils rentrent dans ces écoles, pensent qu'ils ont fait le boulot. Ce n'est pas le cas de tous bien sûr. Mais cette logique-là est inscrite, malheureusement.

\section{E.B. : Alors, quels sont les possibles pour les école nationales de théâtre?}

B.T. : Je ne suis pas en train de dire : supprimons les grandes écoles. Je dis : faisons autrement avec les grandes écoles. Pour revenir à la question de la sélection, je pense que l'équipe ou les équipes pédagogiques de ces écoles devraient se mettre en tâche de sélectionner les jeunes gens là où ils sont. Il faudrait faire le lien avec les conservatoires, les centaines de lieux de pédagogie extraordinaire, qui peuvent être des es petites structures. Quand j'étais au Ministère, la distinction était faite avec 
violence: il y a les écoles nationales et puis, les conservatoires, comme s'ils ne faisaient pas du tout la même chose. Ces gens ne se parlent jamais. Ce sont deux mondes complètement dissociés alors qu'ils devraient être complètement associés. Je pense que la meilleure des sélections pourrait se faire en assistant aux différents travaux des élèves. Pourquoi mettre toute cette énergie à préparer un concours? Alors qu'au fond ils préparent le concours dès qu'ils ont eu un désir de théâtre et que ce désir s'est concrétisé dans le fait d'aller à un atelier au lycée, ou de rassembler trois camarades pour travailler le samedi... On connait bien tout ça. C'est là qu'il faudrait aller voir. Et effectivement, il y aura des choix. Je ne suis pas en train de dire : tout le monde peut être artiste. Non. Le monde de l'art est un monde cruel parce qu'il y a ceux qui en seront et ceux qui n'en seront pas. C'est cruel et binaire mais ça a aussi la force du binaire, c'est-à-dire que c'est évident. Je l'ai toujours vu par expérience dans les jurys.

E.B. : Et dans la pédagogie elle-même, comment intègre-t-on ce droit au rater quand on enseigne en art?

B.T. : Je pense qu'il faut imaginer des écoles qui cessent de vouloir être la réplique du prétendu monde du travail. Il faut oublier cette fausse bonne idée du metteur en scène qui arrive dans une école et qui dit: " On va faire un spectacle comme je fais d'habitude. » Non, justement. Ce serait une tellement belle occasion de ne pas faire comme on fait d'habitude. Quand on arrive dans une école, on devrait se défaire de ses habitudes. Ces habitudes sont très puissantes en tant qu'elles forgent une expérience. Mais je pense que le premier qui devrait sortir de la route balisée, c'est le professeur ou l'artiste qui arrive dans une école et qui devient professeur. Autrement dit, les écoles sont structurées sur le modèle du compagnonnage du $15^{\mathrm{e}}$ siècle. L'artiste arrive, il ne dit pas le mot car le mot est démonétisé, mais il arrive en maitre et il a autour de lui des disciples ou plus exactement des compagnons qu'il associe à un projet de spectacle. Et donc l'enjeu c'est de réussir le spectacle. Sans arrêt dans leur formation, les jeunes gens sont sommés de réussir. Je ne dis pas qu'il faudrait qu'ils ratent tout. Mais je crois qu'il faudrait sortir du couple raté/réussite pour introduire la question du processus. Ce qu'on avait tenté à l'ERAC, c'est qu'un artiste accompagne une promotion sur trois ans. On voit alors apparaître des choses qui ne rentrent pas dans le modèle dominant de la production d'un spectacle. Les élèves auront bien le temps de produire des spectacles. Il y a tellement de possibles à explorer. Il y a tellement d'investigations que l'on peut faire dans une école qui, comme le dit Vitez, est le plus beau plateau du monde, justement parce que c'est un plateau où il n'y a pas d'injonction à la production. Mais l'école doit toutefois convier du public. L'idée de bunkeriser l'école pendant trois ans me paraît problématique. Il faut du public, de la circulation, de l'air qui passe. L'école doit être un lieu ouvert mais ça peut se faire autrement que sous la forme d'un spectacle. Il y a plein d'autres manières d'imaginer un public. On revient à cette notion fondamentale du public comme celui qui peut évaluer ce qui est raté ou réussi. Il serait beaucoup plus intéressant d'inviter quelques jeunes d'une classe d'option théâtre à entrer dans l'école et à passer une après-midi avec les élèves. Il faut que ça cristallise car le théâtre est un rendez-vous. Mais on peut le faire d'une toute autre manière que par la fabrication d'un pseudo-spectacle avec des metteurs en scène très armés qui vont faire comme dans la vraie vie tout en sachant que ce n'est pas la vraie vie.

E.B.: Les cours réguliers qu'ont les élèves en dehors de ces mises en situation professionnelles, sont finalement les cours qui ne sont pas du théâtre, c'est-à-dire les cours 
de chant, de danse, de cirque... Et finalement, le cours où on peut rater puisqu'il n'y a pas de spectacle en préparation n'est donc pas celui qui travaille de front la pratique de l'acteur.

B.T. : Et c'est celui qui n'est pas valorisé. Parce que la rencontre avec le metteur en scène, qui a généralement lieu l'après-midi, est le cœur du travail. Donc les cours du matin sont dévalorisés et désertés. On peut lutter. Je l'ai impulsé à l'ERAC. C'est une école où on peut poser des vraies questions. Comment fait-on face à cette idée que les cours du matin sont vécus comme un boulet par les élèves? Une de mes propositions a été de ne pas suivre de programme pour mes cours du matin mais de me mettre au service des metteurs en scène et artistes qui arrivent dans l'école. À chaque fois la question est d'établir ce que je peux apporter à la personne qui arrive. Dans la plupart des cas on a, à deux, inventé quelque chose. Très concrètement ça veut dire que le metteur en scène vient au cours du matin et que je suis présent dans le travail de l'après-midi. Ça transforme toutes les valeurs. Quand les étudiantes et les étudiants voyaient arriver Hubert Colas ou Ludovic Lagarde pour assister aux lectures du matin, ça changeait tout. Et comme, d'une certaine manière, mon cours continuait l'après-midi, alors il n'était plus un cours du matin. Ce qui est vrai de la dramaturgie est vrai du chant, du tai-chi : pour rendre leur dignité à ces techniques, il faut les intégrer dans le processus du plateau ou en tout cas favoriser le maximum de ponts et passerelles. Alors ça devient un cours à plusieurs mains. Finalement, c'est là qu'on se rapproche le plus de la situation professionnelle. Car lorsqu'un metteur en scène invite un clown ou le traducteur, il veut en tirer le maximum. Quand André Markowicz vient rendre visite aux actrices et aux acteurs qui travaillent sur une de ses traductions de Tchekhov, il est au cœur de la question! Il est au cœur du jeu! Voilà, ce serait une piste pédagogique: cesser de cloisonner les disciplines. C'était aussi ma position avec la revue Mouvement, revue indisciplinaire. Ça ne veut pas dire qu'il n'y a pas de disciplines. Chacune a sa rigueur et sa technicité. Mais il y a un moment où cette technicité doit se diluer dans le projet global.

\section{E.B. : Comment avez-vous intégré cela à votre enseignement ?}

B.T. : Je pense que les étudiantes et les étudiants à qui j'ai apporté quelque chose, ce n'est pas parce qu'ils ont appris plein de choses avec moi. C'est parce que tout ce qu'ils ont appris s'est dilué. Mais ils ont sans doute appris une posture dans la lecture, dans la prise de parole, dans l'écoute. Et ça c'est pour la vie. J'ai toujours dit à mes étudiantes et mes étudiants, le premier jour, que mon seul souhait pour le travail engagé sur trois ans, c'était qu'ils aient un livre dans la poche. Ce désir-là. Le livre est une présence essentielle. L'actrice ou l'acteur lit par définition puisqu'il dit des mots. Et il y a toute cette dualité en eux : moi je suis dans le faire, je ne suis pas dans la théorie. Mais pour pouvoir bien faire ton travail de transmetteur de mots, il faut pouvoir bien lire, il faut que tu te réappropries cette affaire de la lecture qui n'est pas ton ennemie, qui n'est pas hors d'atteinte. La lecture est essentielle et il faut sortir de ce pensum que peut être la lecture à la table. Il faut se rappeler le travail que faisait Gabily avec le groupe T'chan'G ! (1989-1996) dont j'ai eu la chance d'être, comme il disait, l'interlocuteur. Les lectures à la table, c'était du plateau : il se jouait des choses dans ces lectures qui étaient déjà absolument du théâtre et qui d'ailleurs allaient se retrouver dans la création. Donc la lecture, c'est le travail de l'acteur. Il faut faire en sorte qu'il se l'approprie, qu'il apprivoise, qu'il ne la considère pas avec une crainte doublée de respect. "Oui mais moi je suis pas un intello, tu sais bien. » Eh bien si. Si on s'en tient à des distinctions basiques, l'actrice ou l'acteur ne fait pas un travail manuel. L'actrice ou l'acteur fait un travail intellectuel. Il est dans cette catégorie. Ce 
sont des mots qui ne sont pas des gros mots. Ce ne sont pas des mots méprisables. Ce sont des mots qu'il faut se réapproprier. Il faut combler ce fossé terrible entre la théorie et la pratique. D'où l'importance de l'arrivée, enfin, en France de la recherche-création. C'est une piste magnifique pour arriver à ce rapprochement.

E.B. : Et l'espace-temps de cette recherche-création en tant qu'elle fait place aux ratés, ce serait à la fois l'école et la répétition?

B.T. : C'est la longue durée. Il faut se réapproprier le temps long. Il faut en finir avec cette parcellisation du temps. La discontinuité peut avoir des vertus, bien sûr ! On sait bien qu'une actrice ou un acteur après quinze ans avec un metteur en scène peut s'épuiser. Mais il peut aussi partir et revenir. On voit bien avec Tanguy ou Mnouchkine à quel point ça fonctionne. Les actrices et les acteurs partent mais la porte reste ouverte. Même s'ils partent très fâchés.

E.B. : Et si l'on pense maintenant le raté dans la représentation. Est-ce qu'il y a un espace et un temps pour ça?

B.T.: Déjà il faut pouvoir dire que le raté dans un temps $t$ peut devenir la réussite dans un temps $t+$, comme on l'a déjà évoqué pour Le Soulier de Satin dans la mise en scène de Vitez. Et puis on peut aussi considérer la revendication du raté. On peut ne pas vouloir entrer dans ce modèle de réussite. On peut vouloir trouver une alternative. Il y a beaucoup de metteurs en scène qui sont sortis du système qu'on a décrit tout à l'heure comme sélectif et pyramidal. On peut vouloir ne pas être de ce monde-là. Et ça nous amène à Walter Benjamin. Il fait une thèse de doctorat qu'il réussit et enchaîne sur l'habilitation qu'il rate. Mais il y a chez lui une conduite d'échec car il savait très bien que cette seconde thèse n'était pas recevable, non pas parce qu'il n'aurait pas été capable de répondre aux critères du monde académique mais parce que son texte était justement une mise en crise des critères académiques de son époque, à savoir le monde systémique. C'est une dynamite lancée contre le mur compact de l'université allemande et il le sait. On peut donc mener un travail dont on sait pertinemment qu'il va mener au raté. Il dit ça avec une acuité inouïe quand il écrit à Scholem: "cette tentative forcenée de jeter un pont à partir de l'Allemagne pour ma sortie est la dernière que je fais. Si elle échoue il me faudra chercher le salut en nageant, c'est-à-dire me frayer comme je pourrais un chemin à l'étranger ${ }^{3}$.» C'est une phrase incroyable. On entend l'étranger de toutes les manières possibles mais surtout on entend très bien qu'il va devoir fuir l'Allemagne parce qu'il était poursuivi par les nazis. Il sait très bien que sa thèse ne va pas être acceptée et il fait néanmoins le pari qu'elle pourrait l'être. Même si cette thèse d'habilitation est irrecevable, le professeur qui va la lire pourrait tenter de comprendre pourquoi il ne peut pas la recevoir. C'est d'ailleurs ce qui va se passer. Le docteur chargé de lire sa thèse ne comprend rien et il la confie donc à son assistant qui n'est autre que Max Horkheimer. Celui-ci lit le texte et ne comprend rien. Mais il fait le sale boulot et il entérine la sentence. Alors que Horkheimer aurait pu prendre le parti de Benjamin et dire que c'est une thèse complètement hors cadre qui inverse les présupposés. À cette époque, le drame baroque allemand du $18^{\mathrm{e}}$ siècle est complètement dénigré. Donc, déjà, son objet d'étude n'est pas recevable. Et, en plus, il ne le traite pas avec le cadre conceptuel de l'historicisme allemand en faisant des hypothèses conceptuelles qu'il conforterait par son étude. À l'inverse, il dit que ce sont ces textes qui génèrent une nouvelle grille de lecture, ce qu'il appelle une critique. Ce ne sont pas des textes sur 
lesquels on plaque un modèle, ce sont des textes qui génèrent une mise en crise du modèle.

E.B. : Sa logique du rater lui permet d'aller à rebours d'un système et de toute préconception historiciste.

B.T.: Oui. Il s'agit de prendre à rebrousse-poil le système et de laisser parler le réel, en l'occurrence la littérature. Le texte dit ce qu'il y a à penser et non pas ce qu'on veut lui faire dire. C'est le problème de la recherche académique encore aujourd'hui malheureusement: on ne cherche que ce qu'on sait qu'on va trouver. Là, au contraire, il s'expose à l'imprévu et à l'inconnu. Il sait que ça va rater mais il insiste et donc il sort du système, de l'université allemande, de l'Allemagne. Et il cherche un pont mais il n'en trouvera pas beaucoup. J'ai pris cet exemple de la thèse d'habilitation mais Benjamin a, toute sa vie, une conduite d'échec. Il savait très bien que ce qu'il pensait le mènerait à l'échec et il ne cherchait pas à réussir. C'est une posture très forte qui m'amène à évoquer Asja Lacis qui n'est souvent connue que pour être le grand amour de Walter Benjamin. Il y a de fait entre eux un puissant dialogue philosophique, intellectuel et esthétique. Et pourtant rien ne devait les réunir. Lui est un intellectuel de la grande bourgeoisie berlinoise et elle, lettonne, vient d'un milieu très populaire et s'est infiltrée dans le monde de l'art de manière brillantissime. Elle réussit tout. On lui proposera d'ailleurs de diriger le grand théâtre de Riga, ce qu'elle refusera. Le vrai travail, pour elle, n'est pas dans un théâtre. Il est dans la rue avec les enfants, orphelins de guerre. On est dans les années 1920 et le carnage de la première guerre mondiale a laissé des dizaines de milliers d'enfants dans la rue. Ils forment des bandes, volent, violent et terrorisent les populations. Face à ce fléau social qui a lieu de fait dans toute l'Europe, son projet est de ré-humaniser ces gamins en faisant du théâtre avec eux. Elle se frotte à l'institution d'ailleurs. Quand elle arrive à Berlin, elle propose à la fondation Karl Liebknecht un projet pour travailler avec les enfants des rues et elle demande à Benjamin d'écrire la note d'intention. La fondation répond alors qu'on n'y comprend rien. Et tout le monde sait très bien que c'est son amant, Benjamin, qui a écrit le texte. Asja Lacis est très en colère et lui renvoie son texte. C'est une femme qui n'a pas peur de l'autorité. Il reprend donc son texte - on a d'ailleurs perdu la première version. Et la seconde version s'intitule «Programme pour un théâtre d'enfants prolétariens ${ }^{4}$ ». C'est un texte extrêmement puissant mais malheureusement pas très accessible en français. $\mathrm{Si}$ on me demandait de choisir un seul texte sur le théatre du XXe siècle, je choisirais celui-ci. C'est un texte que l'on pourrait donner à tous les élèves d'une école de théâtre. Évidemment le projet d'Asja Lacis va avorter ou rater. Ce qu'elle a pu faire à Moscou ou à Riga n'est pas possible à Berlin car les Allemands ne sont pas prêts. Néanmoins, de ce raté est né ce texte fondamental qui dit que l'espace du plateau n'est pas un espace d'autorité. Il n'y a aucune autorité, ni celle du texte, ni celle du metteur en scène, ni celle du sens, ni celle de l'histoire. Il n'y a aucune autorité sinon celle de ceux qui sont, donc de ceux qui ne savent pas, donc des enfants. C'est un programme théâtral puissant qui vient d'un échec ou plutôt d'un raté.

E.B. : C'est un raté puisqu'il produit quelque chose. L'échec serait une fin tragique et défınitive tandis qu'il y a là une seconde étape. II y a un dépassement de l'échec qui transforme finalement l'échec en raté.

B.T. : Et on peut même anticiper le raté ! On a toujours l'impression que le raté est un événement terrible. De fait, il y a aussi des ratés dont on ne se relève pas. Se planter à Avignon par exemple, c'est terrible. Combien de metteurs en scène ne se sont pas 
relevés d'avoir raté un spectacle à Avignon ? Cela me fait penser au raté monumental du Macbeth de Marc François, un metteur en scène lunaire et fulgurant dans le sillage de Claude Régy. En 1996, il a monté un Macbeth qui postulait en fait que tout ce que disent ces personnages, ce sont leurs rêves. Ils rêvent. Les actrices et les acteurs ont donc travaillé sur leurs rêves et se sont mis en posture de rêver ce spectacle. Et ça allait très loin. Il avait cette radicalité-là. Et ça donnait donc un spectacle complètement lunaire. Il aurait fallu que les spectateurs se mettent eux-mêmes dans la position de rêveurs. On était 800 spectateurs dans la salle Jean Vilar du TNB, peutêtre même plus. Enfin, c'était une salle pleine, une salle de première. Toute la profession théâtrale était là. C'est finalement l'équivalent d'Avignon pendant l'hiver. Nous étions 800 et il restait dix-sept personnes quatre heures et demie plus tard. Je pourrais faire la liste des gens. Il y avait les amis, la famille théâtrale. Je dirais que Marc ne s'en est jamais remis. Ça se termine par un suicide. Il ne s'en est jamais remis et on ne lui a jamais permis de s'en remettre. Autrement dit, on ne lui a pas pardonné. Ça ne pardonne pas, l'échec. On ne pardonne pas aux gens qui échouent parce qu'on y voit un miroir de soi-même.

E.B. : C'est aussi qu'on ne leur permet pas d'en faire un raté finalement. On les condamne à l'échec.

B.T. : Il n'y a pas de circonstances atténuantes. Il n'y a pas de pardon. On n'a jamais permis à Marc François de revenir sur une scène. En plus, c'était un immense acteur. On ne lui a jamais permis de revenir dans la lumière parce qu'il avait été trop loin dans l'échec. Je ne sais pas comment il l'aurait formulé mais il savait très bien que ce n'était pas grand public, pour dire les choses simplement. Évidemment il en a fait le choix. Mais comment a-t-il pu penser que ce travail était possible dans la salle Jean Vilar du TNB ? C'est ça la question. Parce qu'il aurait pu faire le même spectacle dans ce qui était à cette époque la cave du théâtre et où travaillait l'école. Il aurait pu en faire quelque chose de radical pour quinze spectateurs et pas un de plus. Et ça aurait probablement été un succès. Ou au moins on aurait dit : «Il faut y aller ! ». Ça aurait été complètement autre chose.

\section{E.B. : Ça aurait de fait créé une sélection parmi les spectateurs.}

B.T. : Absolument. Pour reprendre les critères de Claude Régy, le spectateur doit se mettre au travail. Il doit être disponible et capable de comprendre pour être de plainpied avec le spectacle. Et ça s'accompagne chez Claude Régy d'une chose qui pour certains était jugée comme extrêmement élitiste : l'idée que l'on ne s'improvise pas spectateur, c'est un processus et pas un droit. Il y a sans doute plein de défauts à cette posture mais ce qu'il y a de vrai, c'est que le jugement sur le théâtre est une chose très complexe. Il n'y a pas de jugement immédiat. Moi je me sens en général incapable de commenter un spectacle dans l'immédiat après-représentation. Et j'ai d'ailleurs souvent usé de la formule de Claude Régy qui disait : « Ne soyons pas plus intelligents qu'on est; après le spectacle, laissons les étoiles et la nuit faire leur travail; nous serons plus intelligents demain matin ». Il y a là quelque chose de très vrai. L'immédiateté de la réponse va jusqu'à produire des excès. «Comment pouvezvous nous imposer ça!». Cette fameuse phrase a été prononcée à Avignon en 2005 par l'actrice Hervée de Lafond ${ }^{5}$. Elle a interrompu comme ça un spectacle de Pascal Rambert (After/Before). C'était un travail sur le vieillissement et sur l'usure où les acteurs n'étaient pas des acteurs et les corps n'étaient pas formatés. Il y avait des corps vieux. Le spectacle consistait à répéter une séquence musicale et rythmique 
trois fois sous des modalités différentes. La variation est une chose que la musique connaît bien. La boucle initiale était déjà malaisante, âpre et rude quoique fluide et elle était donc répétée trois fois. Et, dans la répétition, il y a de l'insupportable. Le bégaiement par exemple est difficile à accepter. La reprise n'est pas du tout théâtrale : l'acteur doit être fluide, il doit déclamer, articuler. Donc, Pascal Rambert posait un geste, une recherche, qui là aussi n'étaient pas pour Avignon à trois heures de l'après-midi. Soudain, Hervée de Lafond se lève et dit cette phrase : "Comment pouvez-vous nous imposer ça!» Cette phrase met le feu aux poudres et, en trois jours, tout le monde répète en boucle cette idée que ça n'est plus possible avec toute sorte de variations.

E.B. : Ça pose à nouveau la question du jugement. Qui peut juger du raté d'un spectacle et quand?

B.T.: Oui. Il y a eu une sorte d'hypertrophie du jugement où tout le monde se permettait de donner son avis, tout le temps. Je me souviens d'un Monsieur, à un spectacle de Gisèle Vienne (Une belle enfant blonde), qui s'adresse à Catherine RobbeGrillet et qui lui dit : «On a l'impression d'être à la télé, là !» Et, effectivement, il est devant la télé, il mange des chips, il dit ce qui le traverse, il zappe. Donc tout le monde juge sans arrêt comme si on avait, au théâtre, ce système de vote en temps réel de la télévision. Je donne ces exemples car il y apparaît que ce qui fait le raté c'est souvent une précipitation du jugement. Ce n'est pas simple d'être spectateur et d'émettre un jugement critique, qu'on soit critique ou spectateur lambda d'ailleurs. C'est d'ailleurs pour ça que beaucoup de gens ne vont pas au théâtre. Mais, de fait, il y a à Avignon un public nombreux et divers - fort heureusement. Néanmoins ça pose la question de mettre n'importe qui devant n'importe quoi. Comment fait-on? À l'évidence, la multiplication des propositions d'Avignon fait que les travaux que je viens de citer sont finalement juxtaposés à Bartabas et Ariane Mnouchkine. Et la question se pose d'un certain fléchage. Il me semble que le premier des fléchages, c'est le lieu. Et, à Avignon, se pose particulièrement la question de où on fait théâtre. On peut faire théâtre de tout, certes, mais où et comment? En fait, ces spectacles-là, devant ces spectateurs-là produisent l'explosion, ce que j'appelle, moi, le scandale.

Le scandale, c'est la rupture du pacte de fiction. À un moment donné, on n'accepte plus la fiction qui nous est racontée, on ramène le réel et on rompt ce pacte. La question ensuite, c'est: est-ce que cette rupture-là pour autant suppose que le spectacle est raté ? Dans le cas de Pascal Rambert, c'est ce qu'on a dit : il est sur des territoires complètement confus, c'est n'importe quoi. Mais ce même Pascal Rambert, dix ans plus tard, écrit Clôture de l'amour qui est déjà un classique, que les professeurs de lycée n'ont aucun mal à faire lire à leurs élèves, qui tourne dans le monde entier, qui est traduit dans cinquante langues. Par là je nomme les critères d'une réussite. Et c'est la même personne qui a raté à Avignon en 2005, participant à déclencher la fameuse querelle d'Avignon, dont j'ai d'ailleurs été un des acteurs avec le petit livre ${ }^{6}$ écrit à chaud avec Georges Banu, juste après l'été. Donc Rambert, selon mon analyse, est celui qui génère le scandale par son ratage et, dix ans plus tard il crée une écriture théâtrale qui est déjà anthologique. C'est intéressant cette dialectique.

E.B. : Ça indique aussi qu'une histoire des ratés ne peut pas être linéaire. Elle est forcément antihistoriciste.

B.T. : Elle oblige à accepter le fragment, la rupture. Simplement, l'œuvre n'est pas forcément recevable dans le temps où elle éclot. C'est vraiment le principe du 
critique littéraire qu'est Benjamin. Ces drames baroques allemands mineurs du 18e siècle, sont complètement inaudibles et irrecevables. Et soudain, après trois siècles, ils reviennent dans la lumière. C'est vrai parce que Benjamin en a fait une thèse qui est restée célèbre mais c'est à nuancer parce que ce n'est pas quelqu'un comme Ostermeier qui s'en empare. Le principe est toutefois extrêmement valide. Combien d'œuvres ne sont pas audibles au moment de leur éclosion? Ça va donner la figure de l'artiste maudit et ça donne aussi cette tradition du mineur que Gilles Deleuze développe avec Félix Guattari dans Kafka, Pour une littérature mineure ${ }^{7}$. On peut passer sa vie d'artiste à être mineur et à être pourtant essentiel.

E.B. : Ça rejoint ce que vous disiez de la réception d'un spectacle par le critique comme par le spectateur. La réception de l'œuvre d'art engage forcément une éthique. Et cette éthique de la réception supposerait de faire une place aux ratés.

B.T. : Effectivement. Pour reprendre un thème rebattu, il faut une éducation, une formation. Mais ces mots font vite moralistes.

\section{E.B. : Une expérience ?}

B.T.: Oui. Si on prend par exemple ma situation, je vis en montagne et cela suppose une accoutumance déjà à l'altitude. Comment est-ce qu'on s'accoutume? Comment développe-t-on des techniques ? Comment apprend-on ce qu'on peut faire et ce qu'on ne peut pas faire? Comment ne pas prendre de risque? Ça prend du temps. C'est une sorte de formation. En tout cas, on doit accepter d'être modelé par cette montagne qui nous entoure et qui est tellement énigmatique. D'une certaine façon, c'est pareil dans le monde du théâtre qui est bien énigmatique. On n'arrive ni vierge, ni formé. Je crois que c'est une des raisons de la querelle d'Avignon en 2005. La plupart des critiques qui avaient des rubriques culture et qui avaient le pouvoir de décider ce qu'on met en une, ce qu'on va privilégier et comment on va le présenter, n'étaient pas capables de regarder les spectacles proposés même s'ils avaient trente ans d'expérience. En effet, il y a eu une rupture importante. On était passé de l'ère des metteurs en scène à l'ère des écrivains de plateau, dans mon lexique. Pour le dire autrement, on était passé d'un régime où le théâtre était déterminé par le texte, fût-il médié par le metteur en scène et son interprétation, à un régime où le texte n'était autre que le plateau et où faisaient donc écriture plein de choses qui, pour un critique de 60 ans, n'étaient pas appréhendables. Ils étaient tout simplement perdus, comme on peut se perdre en montagne. C'est un risque terrible, se perdre en montagne et être pris par la nuit. Ils étaient, en fait, pris par la nuit. Un nouveau théâtre se développait, une nouvelle génération, plus tout à fait jeune. Jan Fabre, par exemple, n'était pas un nouveau venu. Néanmoins, beaucoup d'observateurs du champ théâtral n'étaient pas équipés. Ils n'avaient pas les bonnes chaussures, pour filer la métaphore.

\section{E.B. : On peut revenir, pour conclure, sur le droit au rater?}

B.T. : Il y a une notion qui me vient. Le droit à l'essai. C'est une chose qui nous vient du cinéma qui est pourtant un art incroyablement pris dans une logique marchande de compétitivité. Mais le cinéma a inventé cette belle notion d'art et essai. Le droit à l'essai pourrait être une issue. Car le raté est une notion très morale. «C'est un raté ». «Il est le raté de la famille».

\section{E.B. : C'est moral parce que ça engage le jugement.}

B.T. : C'est à partir d'un arsenal moral qu'on énonce un point de vue. Et toute morale, quand elle s'impose, donne le droit à l'existence ou non. C'est un raté, parce qu'il n'a 
pas réussi à s'insérer dans le monde du travail, parce qu'il a pris de la drogue, parce qu'il était hippie. Donc, cette formule dépend de la notion de travail. Mais on peut tout à fait inverser les rapports. On en est venu à penser que l'oisiveté est ce qu'il ne fallait pas faire et qu'un homme actif et vital dans la société capitaliste est un homme qui travaille et produit. Or, dans le monde romain, l'otium, c'est-à-dire, la non production est précisément la valeur de distinction du citoyen. Et par opposition, le négoce, le negotium, en est la négation. Le travail comme valeur suprême est donc arbitraire. L'otium peut dès lors être une posture dans l'existence. Il s'agirait de penser ce paradoxe : l'oisiveté n'est pas le «ne rien faire ». On peut parfaitement, en n'étant pas pris dans l'emploi salarié, avoir plein de travail. Ce n'est pas binaire. Le travail salarié est l'alpha et l'oméga de notre société mais ça n'épuise pas l'ensemble du champ du travail. Il faut démoraliser un certain nombre de nos concepts car ces mots-là sont très puissants.

\section{E.B. : Alors, revendiquer le rater serait revendiquer une éthique anticonformiste.}

B.T.: Plutôt une éthique qui valorise des choses qui ne sont pas valorisées dans l'éthique dominante. Il s'agit d'exposer le système, car dans celui-ci, le travail, reste le tripalium, la torture. Combien de personnes me disent, alors que le confinement touche à sa fin en France : «J'appréhende de retourner travailler. Comme j'étais bien chez moi!» Pendant le confinement, il y a eu une surcharge: le télétravail, les enfants... Mais ça a aussi libéré un espace autre, un espace de possibles, un espace où on peut oser et même se perdre. Accepter de rater c'est aussi accepter de se perdre, de quitter les chemins balisés, de ne pas avoir d'objectif. Et cela passe par une réappropriation du chez-soi.

\section{E.B. : On en revient à l'espace-temps du rater.}

B.T. : Oui. Je pense que le monde culturel s'est tellement structuré qu'il est devenu hors sol. Aujourd'hui, ce qu'on voit apparaître, c'est une volonté de se ré-ancrer dans les territoires et de se réapproprier sa maison, fût-elle éphémère. On peut reprendre le couple de notions issu d'une conversation entre Brecht et Benjamin's. Il y a deux façons d'habiter : dans la première, on modèle l'espace, ou on se l'approprie et dans la deuxième, on se laisse modeler. L'exemple de Benjamin, c'est la logeuse : on loue une chambre chez quelqu'un et, à l'époque, il y avait aussi le couvert. Dans ce cas, on se plie à des horaires, à des habitudes qui ne sont pas les nôtres mais celles de l'autre. Ce sont deux manières d'occuper un lieu. Ça me paraît extrêmement important aujourd'hui. On voit de plus en plus de gens dire : «J'ai envie de travailler depuis chez moi. » Et dans le monde de la culture, cela désigne, un territoire, une région, un village, une périphérie... Cela revient à ce que j'avais développé, il y a quelques années, dans un texte intitulé "Maisons d'artistes" ${ }^{9}$. Les artistes font leur travail dans un espace-temps donné. Et les grandes expériences artistiques ont toujours eu lieu quand il y avait un lieu et un temps : la cave de Kantor, le Piccolo Teatro pour Strehler, la fonderie pour Le théâtre du Radeau. Chaque fois, il s'agit d'une maison.

\section{E.B. : La maison donne l'espace-temps quand le théâtre ne donnerait que l'espace ?}

B.T. : Exactement. Parce que dans une maison, on a les clés. C'est ce que dit Mnouchkine aux nouvelles générations : «Demandez les clés. Si vous n'avez pas les clés, vous êtes foutus. Et prenez garde à les garder!»Vassiliev, quand il venait travailler à l'ENSATT, il offrait une bouteille de vodka au gardien pour pouvoir travailler toute la nuit. Il avait obtenu officiellement de travailler jusqu'à deux heures du matin mais, avec la vodka, il pouvait rester toute la nuit. Ce qui nous ramène à 
l'idée que la question centrale, c'est la question du temps. Dans cette crise que nous traversons et qui est dramatique, on observe toutefois aussi une réappropriation du temps. Je pense très simplement que ce que le capitalisme nous vole le plus, c'est le temps. D'ailleurs, la phrase la plus employée par la classe moyenne blanche occidentale c'est "je suis très occupé ». Moi je ne peux pas ne pas entendre dans «occupé » le terme «occupation». Nous vivons sous un régime d'occupation. Donc, désoccupons-nous!

E.B. : Quand il y a le temps, on a droit au rater puisqu'il n'y a pas d'impératif de réussite pour demain ou même pour après-demain. On peut voir venir.

B.T.: Oui mais il ne faut pas cependant se passer des échéances. Simplement, ces échéances doivent rester une décision de l'artiste. Il est traditionnel dans les grands théâtres russes de reporter la première. On joue quand c'est prêt. Et ce n'est pas un problème parce que la programmation repose sur un répertoire. Donc on peut reporter. Et ce n'est pas parce que l'artiste est un enfant capricieux. C'est parce qu'il a ses règles. C'est ce qui a produit le scandale à la Comédie Française avec Vassiliev (Le Bal Masqué de Mikhail Lermontov, 1992) : il n'a pas pu imposer cette règle. Il savait que le spectacle n'était pas prêt mais il n'a pas pu avoir quinze jours de plus. Il l'a d'ailleurs dit quand les spectateurs ont commencé à arracher les fauteuils : « Revenez dans quinze jours. » En faisant cela, il a repris la main et énoncé sa règle. C'était le premier à dire que, ce jour-là, le spectacle était raté. C'est important de finir làdessus : au théâtre, on rejoue le même spectacle tous les soirs mais on le remet en jeu tous les soirs. On n'est jamais dans du définitif. Tous les jours, ça se rejoue. On le dit souvent à Avignon : le spectacle sera prêt à la fin du festival. C'est un paradoxe absolu puisque des gens du monde entier viennent à Avignon.

E.B. : Est-ce que ça existe, à l'inverse, un spectacle qui serait réussi au début de sa tournée et raté à la fin?

B.T. : C'est l'usure d'un spectacle, oui. C'est d'ailleurs ce qu'on reproche aux Russes. C'est très bien huilé mais ça fabrique la routine. Tout spectacle qui tourne longtemps est menacé de ça, d'être sur des rails et que tout soit calculé. J'ai connu un acteur qui avait calculé que s'il gagnait cinq minutes sur la durée du spectacle, il pourrait prendre le train et rentrer le soir même à Paris. Et il a imposé à ses partenaires de jouer plus vite. Je ne dirai pas de nom! La routine, c'est le poison du théâtre. L'antidote, c'est d'embrasser l'imprévisible et de se remettre en jeu et en question tous les soirs. C'est très difficile.

\section{E.B. : Ce qu'on vient voir, c'est la possibilité du raté.}

B.T. : Ce qu'on vient voir, je crois, c'est la possibilité de la mort de l'acteur. C'est un des ressorts implicites de l'aller au théâtre. À l'inverse, Ariane Mnouchkine disait à ses acteurs de prendre en compte qu'il y a des spectateurs qui vont pour la première fois au théâtre mais qu'il y en a aussi pour qui c'est la dernière fois. Donc bien sûr, le théâtre a un rapport à la mort. Au cirque, le raté c'est la mort. On est loin de la notion d'essai. Autrement dit, on ne peut pas se rater. Au moins dans certaines pratiques, dans l'aérien par exemple, l'artiste de cirque n'a pas le droit au raté. Et, en contrepoint, le clown ne travaille que sur le raté. Je pense que c'est une leçon et une école extraordinaires pour l'acteur. Il a besoin de penser, au plus profond de lui-même, qu'il est un clown, c'est-à-dire quelqu'un qui rate. Parce que le rater ce n'est pas qu'un événement, c'est un mode d'être et de créer. 


\section{BIBLIOGRAPHIE}

BANU, Georges et TACKELS, Bruno, Le cas Avignon 2005 : regards critiques, Vic-la-Gardiole,

l'Entretemps éd., 2005.

BENJAMIN, Walter, Correspondance (1910-1928), t. 1, Paris, Aubier-Montaigne, 1979.

BENJAMIN, Walter, Essais sur Brecht, traduit par Philippe Ivernel, Paris, La Fabrique Éditions, 2003.

DELEUZE, Gilles et GUATTARI, Félix, Kafka, Pour une littérature mineure, Paris, Éditions de Minuit, 1975.

\section{NOTES}

1. https://www.compagniedesindes.tv

2. Bruno Tackels fait ici référence au Bal Masqué de Mikhail Lermontov qu'Anatoli Vassiliev monte à la Comédie-Française en 1992.

3. Walter Benjamin, Correspondance (1910-1928), t. 1, Paris, Aubier-Montaigne, 1979, p. 277.

4. Le texte original est disponible ici: https://www.documenta14.de/de/south/

25225_zeichen_einer_anderen_zeit_das_proletarische_theater_als_staette_der_erziehung_texte_von_asja_l_cis_und_walter_benjamin

5. Co-directrice du théâtre de l'Unité.

6. Georges Banu et Bruno Tackels, Le cas Avignon 2005 : regards critiques, l'Entretemps éd., Vic-laGardiole, 2005.

7. Gilles Deleuze et Félix Guattari, Kafka, Pour une littérature mineure, Éditions de Minuit, Paris, 1975.

8. Walter Benjamin, Essais sur Brecht, traduit par Philippe Ivernel, La Fabrique Éditions, Paris 2003, p. 166-204.

9. Ce texte a été publié par fragments dans la revue Mouvement et est à paraître dans un essai en dialogue avec Alain Badiou.

\section{RÉSUMÉS}

Cet entretien explore la question du rater au théâtre. Bruno Tackels y développe d'abord l'idée du rendez-vous manqué et relie ainsi la question à l'essence du théâtre qu'il confronte ensuite aux politiques culturelles. Il insiste en particulier sur la manière dont les processus de sélection engendrent du ratage programmé, ce qui le conduit à poser la question du jugement : qui a le droit de décider que c'est raté ? Qui fabrique l'histoire des succès au théâtre ? La fin de l'entretien propose alors de dépasser le couple raté/réussite et envisage le rater comme processus dans la pédagogie, la création et la pensée du théâtre. 


\section{INDEX}

Mots-clés : histoire du théâtre, processus de sélection, mise en scène contemporaine, Benjamin (Walter), Lacis (Asja), Lambert (Pascal), Vassiliev (Anatoli), Vienne (Gisèle), Vitez (Antoine)

\section{AUTEURS}

\section{BRUNO TACKELS}

Bruno Tackels est dramaturge et essayiste. Il est agrégé et docteur en philosophie et a enseigné en France à l'université et dans les écoles nationales de théâtre. Il a publié trois livres consacrés à Walter Benjamin et notamment sa biographie (Walter Benjamin, une vie dans les textes, 2009). Il a été le rédacteur de la revue Mouvement et a écrit une série d'ouvrages consacrés aux écritures et aux écrivains de plateau publiée par les Solitaires Intempestifs (2005-2015). Après avoir passé des années au cœur de la création théâtrale française, Bruno Tackels vit aujourd'hui en Colombie où il se consacre à l'écriture et à la culture des oliviers.

\section{ESTELLE BAUDOU}

Pendant la préparation de ce numéro (2019-2021), Estelle Baudou bénéficiait d'un financement Marie Sklodowska Curie de la commission européenne pour mener sa recherche post-doctorale à l'APGRD (Archive of Performances of Greek and Roman Drama) de l'Université d'Oxford (her project has received funding from the European Union's Horizon 2020 research and innovation programme under the Marie Sklodowska-Curie grant agreement No 839770). Son travail, entre pratique et théorie, porte sur la mise en scène de la guerre et des mythes antiques en Europe depuis 1945. Elle a, auparavant, réalisé un doctorat sous la direction de Christian Biet à l'Université de Paris Nanterre (Une Archéologie du commun, Classiques Garnier, 2021). Elle enseigne aussi la dramaturgie au Conservatoire Régional de Paris et travaille à plusieurs spectacles comme dramaturge ou metteure en scène. 